\title{
A Study of the Dynamics of Cardiac Ischemia using Experimental and Modeling Approaches
}

\author{
Shibaji Shome, Jeroen G. Stinstra, Bruce Hopenfeld, Bonnie B. Punske, and Rob S. Macleod \\ CardioVascular Research and Training Institute, University of Utah, Salt Lake City, USA \\ Scientific Computing and Imaging Institute, University of Utah, Salt Lake City, USA \\ Department of Bioengineering, University of Utah, Salt Lake City, USA
}

\begin{abstract}
The dynamics of cardiac ischemia was investigated using experimental studies and computer simulations. An experimental model consisting of an isolated and perfused canine heart with full control over blood flow rate to a targeted coronary artery was used in the experimental study and a realistically shaped computer model of a canine heart, incorporating anisotropic conductivity and realistic fiber orientation, was used in the simulation study. The phenomena investigated were: (1) the influence of fiber rotation on the epicardial potentials during ischemia and (2) the effect of conductivity changes during a period of sustained ischemia. Comparison of preliminary experimental and computer simulation results suggest that as the ischemic region grows from the endocardium towards the epicardium, the epicardial potential patterns follow the rotating fiber orientation in the myocardium. Secondly, in the experimental studies it was observed that prolonged ischemia caused a subsequent reduction in the magnitude of epicardial potentials. Similar results were obtained from the computer model when the conductivity of the tissue in the ischemic region was reduced.
\end{abstract}

Keywords-Cardiac modeling, conductivity, epicardial measurements, fiber orientation, ischemia dynamics.

\section{INTRODUCTION}

A recent modeling study using a full anisotropic model of the heart during ischemia suggests that epicardial potential distributions depend on the underlying fiber structure of the myocardium [1], [2]. Not only do the epicardial potentials depend on macroscopic structures like fiber orientation, studies of changes in the cardiac conductivity suggest that dynamic changes at a cellular level affect this conductivity and thence the electrical properties of the myocardium as a whole [3]. In order to investigate these phenomena predicted by the modeling studies, we present a comparative study of epicardial potential distributions measured during episodes of ischemia with those of the models.

In both the modeling study as well as the experimental study we were interested in the influence of fiber orientation on surface potential distributions during ischemia. Ischemia is a condition that results due to an incompletely compensated increase in metabolic demand or a critical reduction in blood supply or a combination of the two. The presence of ischemia is normally detected by changes in the ST-interval in the electrocardiogram. During this interval, the ischemic cardiac cells have a lower transmembrane potential than the surrounding healthy cells, which leads to injury currents flowing from the healthy zone into the ischemic zone through the intracellular space and returning through the extracellular space. Due to the anisotropic nature of cardiac tissue, these currents flow more easily along the longitudinal fiber direction than the transverse fiber direction. Hence, the injury currents and the epicardial potential distributions depend on the fiber orientation. From a viewpoint outside the heart, the transmural fiber orientation in the left ventricle undergoes a counterclockwise rotation from the epicardium towards endocardium [4]. Epicardial potential mapping studies, where potential maps were obtained by pacing at different transmural locations in the myocardium, show this rotation of the myofibers [5]. A second observation of the bioelectric currents arising in ischemia is that they are strongest at the border zone between healthy and ischemic tissue. Therefore, we hypothesize that as the ischemic area grows from the subendocardial region towards the epicardium, the injury currents would preferentially follow the fiber direction in the ischemic boundary closest to the epicardium. Since the fiber direction rotates transmurally, a growing ischemic region would manifest a similar rotation of the epicardial potential patterns.

Furthermore, one of the cellular effects in ischemia is a decrease of the $\mathrm{pH}$ of the intracellular fluid, a change that is likely to affect the function of the gap junctions [6]. When the $\mathrm{pH}$ decreases, gap junctions begin to uncouple which increases the resistivity between adjacent myocytes and thus reduces the strength of the injury current. Thus, we hypothesize that prolonging ischemia results in the reduction in magnitude of the ST-segment shifts detectable on the epicardium.

\section{METHODS}

\section{A. Simulation Setup}

In order to simulate both the effects of fiber orientation and changes in the effective conductivity, we developed a multi-scale modeling approach [1]-[3] . At a cellular level, the model simulates a piece of cardiac tissue and includes details such as cell geometry, gap junctions, and the specific conductivities of various electrolytes. With this model, one can compute the effective conductivity tensors of a piece of cardiac tissue, which subsequently serve as parameters in a whole heart model. The model of the whole heart simulates epicardial potentials from ischemic sources and is based on the bidomain equations. The anatomical and fiber structure data underlying this model originated in the Auckland canine heart [4] and the ischemic source was a difference in the transmembrane potential between healthy and ischemic tissue of $30 \mathrm{mV}$ [1]. 


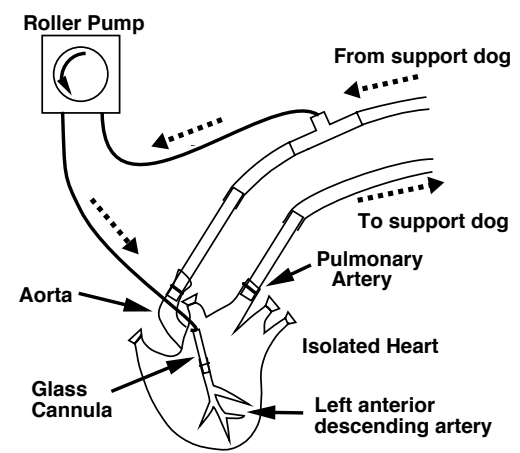

Fig. 1. Schematic of the cannulated isolated heart preparation. Dashed arrows indicate direction of blood flow

\section{B. Experimental Setup}

The details of the preparation have been described previously [7]. Briefly, it consists of an isolated heart, extracted from a dog (weight 15-20 kg) and perfused with blood from a support dog (weight $30-35 \mathrm{~kg}$ ) via a modified Langendorff setup. The blood from the support dog enters the isolated heart via cannulation of the aorta and the circulation loop is completed by returning the blood, from the right ventricle of the isolated heart, via a mechanical pump to the support dog. We suspended the perfused heart in a human-shaped electrolytic tank that was instrumented with 384 surface electrodes.

In a second setup, heart perfusion was by means of a closed circuit oxygenation system enclosed in a warmed $\left(37^{\circ} \mathrm{C}\right)$ and humidified chamber. A mixture of $\mathrm{O}_{2}$ and $\mathrm{CO}_{2}$ was used to maintain the $\mathrm{pH}$ of the perfusate within physiological limits. To control coronary blood flow, we cannulated the left anterior descending coronary artery (LAD) and perfused it by means of a digitial rotary pump (see Fig. 1). The heart rate of the isolated heart was set by atrial and ventricular pacing. We sampled the epicardial potentials by means of a 490 electrode sock covering the ventricles of the isolated heart and a custombuilt, 1024-channel, $1 \mathrm{kHz}$ recording system.

To test the hypothesis that a growing ischemic region will result in rotation of the epicardial potential pattern, we analyzed data from two experiments in which we reduced the coronary flow for fixed intervals and recorded at the end of each interval. In both experiments, the normal flow rate was $34 \mathrm{ml} / \mathrm{min}$ and subsequent flow rates were reduced in steps of $3 \mathrm{ml} / \mathrm{min}$ for 2 minutes each until a flow of $1.3 \mathrm{ml} / \mathrm{min}$ was reached.

To investigate the relationship between sustained ischemia and changes in the conductivity, we analyzed data from an experiment in which we subjected the isolated heart to very low flow conditions $(10 \mathrm{ml} / \mathrm{min})$ for 24 minutes and recorded every 3 minutes within this period. Specifically, the flow rate was set to $10 \mathrm{ml} / \mathrm{min}$ and the flow rate was maintained for 24 minutes with epicardial data recorded every 3 minutes.

From each recording we selected a representative beat for further processing. Post processing consisted of calibration, compensating for baseline drift with a linear correction, and interactive visualization of the results, all with custom software.

\section{RESULTS}

Fig. 2 shows sample results from 3 different experiments and the corresponding simulation studies. Although there were differences between the experiments and simulations in terms of the heart geometry, the specific size and location of the ischemic zone, and the presence of ventricular blood, the goal of the analysis was to compare essential features of the potential distributions.

\section{A. Influence of fiber orientation on epicardial potential pat- terns}

Panel A of Fig. 2 shows the results from an experimental study with stepwise reduction in coronary flow and simulations of stepwise increasing extent of the ischemic zone. The experimental data show the relatively stable potential distribution recorded $40 \mathrm{~ms}$ after the $\mathrm{J}$ point for 5 different levels of perfusion via the coronary catheter inserted at the location of the solid line across the LAD. The simulation results show the potentials for 3 different transmural extents of ischemia, where $100 \%$ indicates fully transmural ischemia. The fourth map in the simulation study shows the constant lateral dimensions of the simulated ischemic zone. The location of the ischemic region in the computer model was such that it mimicked the probable location of ischemia in the experiment as closely as possible. We obtained the fiber orientation direction presented in Panel D of Fig. 2 using the techniques described by Nielsen et al. [4].

A dashed line indicates the direction of the steepest gradient in Panel A and an important finding, common to both sets of results, is the rotation of this line during progression of the extent of ischemic tissue. One can also see the presence of two regions of potential depression flanking a single region of ST-elevation all aligned along the steepest gradient. The results shown in panel $\mathrm{A}$ indicate that as the flow is progressive reduced, the direction of the steepest gradient rotates in a clockwise manner. The extent of this rotation is dependent on both the flow rate and the time for which the low flow rate is maintained. It is known that the subendocardium is the first region affected by compromised flow [8]. As the flow rate is further reduced and maintained, the ischemic area begins to grow towards the epicardium. The pattern of the voltage gradient depends on the fiber directions so that rotation of the fiber direction will also cause rotation of the direction of steepest descent on the epicardium. The simulation results in the lower row of Panel A show a similar, clockwise rotation of the gradient axis.

Panel B contains results from experiments and simulations to show the effect of increasing ischemic extent on the amplitude of epicardial potentials, in this case for an ischemic zone near the apex. In this case, the dominant feature of the maps is a region of elevated potential, this time without neighboring regions of depression, that becomes larger in amplitude and slightly more focused as the ischemia progresses in extent 

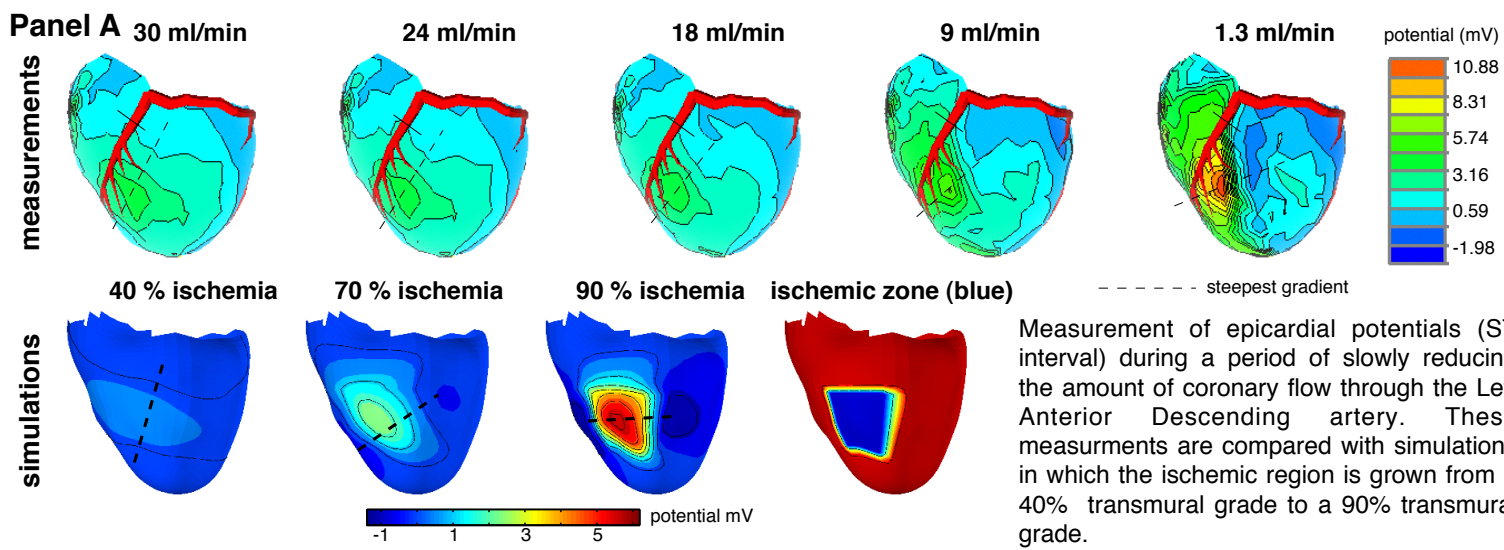

Measurement of epicardial potentials (ST interval) during a period of slowly reducing the amount of coronary flow through the Left Anterior Descending artery. These measurments are compared with simulations in which the ischemic region is grown from a $40 \%$ transmural grade to a $90 \%$ transmural grade.

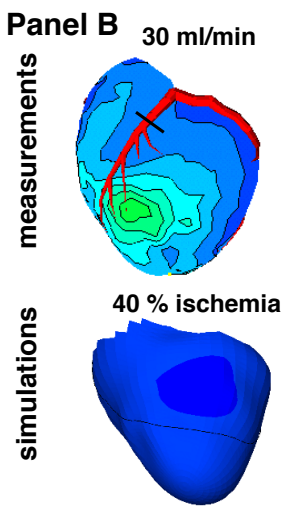

Panel C

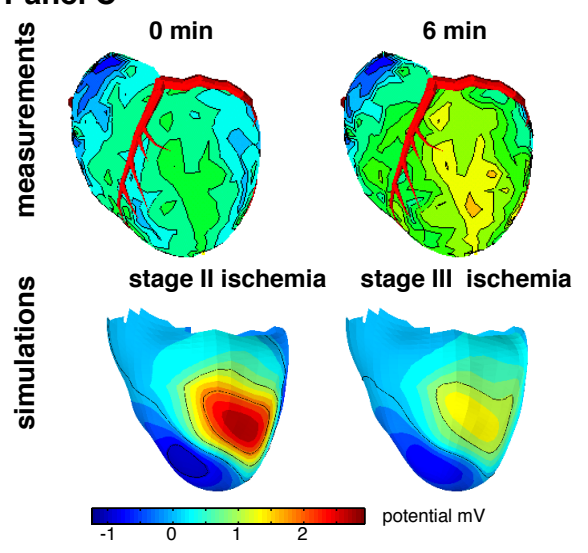

Panel D
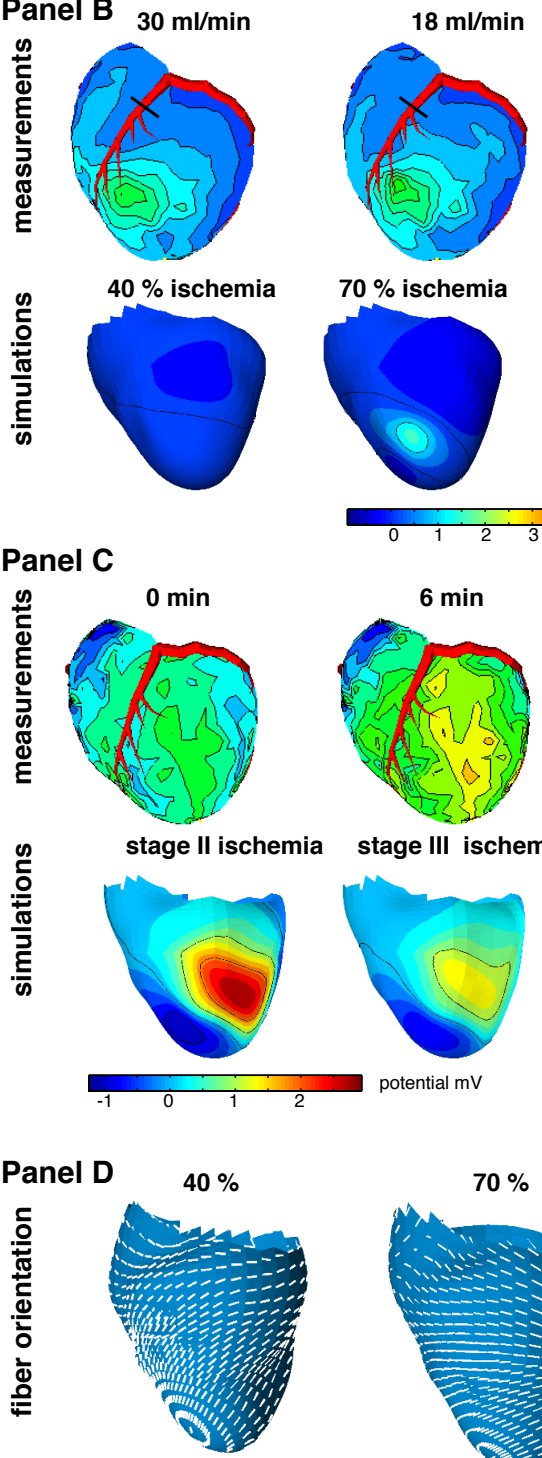
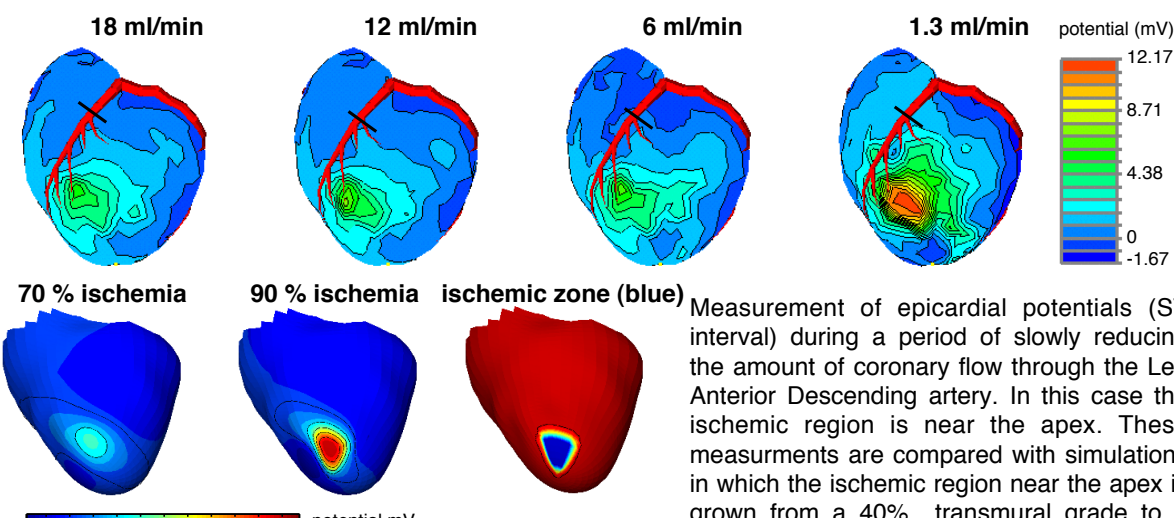

Measurement of epicardial potentials (ST interval) during a period of slowly reducing the amount of coronary flow through the Left Anterior Descending artery. In this case the ischemic region is near the apex. These measurments are compared with simulations in which the ischemic region near the apex is grown from a $40 \%$ transmural grade to a $90 \%$ transmural grade.
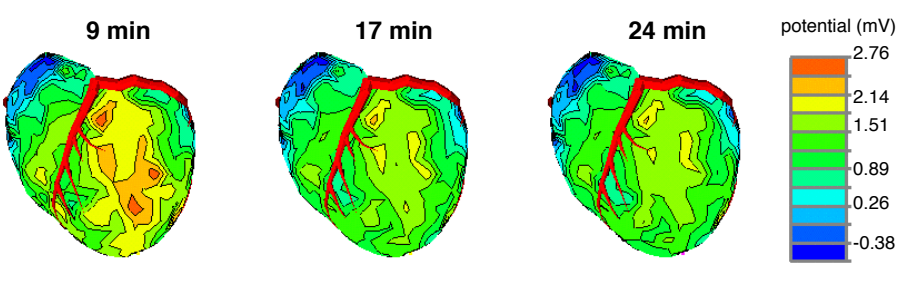

Measurement of epicardial potentials (ST interval) during a sustained period of low flow through the Left Anterior Descending artery $(10 \mathrm{ml} / \mathrm{min})$. Because the reduction in ST elevation after about 15 minutes is attributed to a closure of the gap junctions. these measurments are compared with a simulation in which the conductivity of the ischemic area is altered to reflect the transition from stage II to stage III ischemia. In the underlying model most of the gap junctions were closed leading to an increase of the intracellular resistivity. The simulations depicted here represent a $70 \%$ grade of ischemia in a zone affected by the Left Anterior Descending artery.

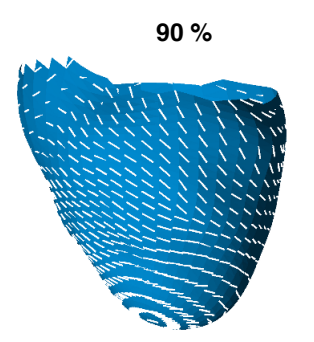

The fiber orientation in the model at different locations within the cardiac wall. The percentage indicates how deep the depicted shell is located within the myocardium $(0 \%$ is the endocardium and $100 \%$ is the epicardium). The white rods indicate the local fiber orientation.

Fig. 2. Comparison between experimental and computer simulation results. 
from endocardium to epicardium. The lack of neighboring depression may arise because of the smaller lateral extent of the ischemic zone (indicated in the last image in the lower row of Panel B) or because of its location closer to the apex, a region in which fiber orientation is more vertical that in the case shown in Panel A.

\section{B. Effect of dynamic change in tissue conductivity during prolonged ischemia}

In panel $\mathrm{C}$ we present the experimental and modeling results obtained from sustained ischemia of a fixed spatial extent. We maintained a flow rate of $10 \mathrm{ml} / \mathrm{min}$ and assumed that this flow rate would be adequate to maintain a viable layer of tissue close to the epicardium but cause ischemia in the endocardial and midmyocardial regions. In the simulation studies, we assigned the ischemic region to extend to about $70 \%$ of the wall thickness from the endocardium. In the experimental results we observed that the ST segment elevation magnitude was highest (about 2-3 mV) around the 9th minute of recording, and that this magnitude progressively reduced and reached a value of around $1-1.5 \mathrm{mV}$ by the 24th minute of reduced flow. Other investigators have shown that gap junctions close between 15 and 25 minutes after onset of ischemia, which causes a reduction in the observed potentials due to reduced conductivity [6]. Stinstra et al. [3] simulated these changes and obtained values for the resulting drop in conductivity. We incorporated these conductivity values in our simulations studies and obtained epicardial pattern distributions before and after gap junction closure (stage II and stage III ischemia in the lower row of Panel C). Comparison of the experiment and simulation results shows good agreement and suggests that progressive decoupling of gap junctions is a viable mechanism for the reduction in ST-segment change that has long been observed clinically.

\section{CONCLUSIONS}

In a previous study of subendocardial ischemia in a canine model, Guyton et al. concluded that the absence of epicardial ST-elevation does not guarantee the absence of ischemia in the subendocardium [9]. We reproduced a similar effect in our modeling study in which ischemia in the subendocardium led to only small elevations of the epicardial potentials. We therefore conclude that since the ischemic zone is far away from the epicardium and the blood in the ventricles forms a low resistance shunt for the injury current, there is not enough current reaching the surface to produce the necessary potential drops. In addition, our results show that the rotation of the epicardial ST-segment potential distributions is indicative of a transmurally growing region of ischemia. Furthermore, we conclude that although epicardial ST segment depression may not fully localize ischemia, the gradient between the ST-elevation and the ST-depression regions is indicative of the transmural depth of the ischemic region. From our experimental results, we see that sustained reduced flow result in epicardial potential patterns that were reproduced by decreasing the conductivity of the the ischemic zone in the computer model. This seems to suggest that changes in conductivity influences epicardial potential patterns and supports the findings of Smith et al. [6]. We demonstrate that the use of an experimental model in conjunction with a simulation study leads to a new insight into the electrophysiological consequences of myocardial ischemia. The clinical implications of these findings motivate further investigations of the phenomena observed through refinement of the modeling and experimental methods.

\section{ACKNOWLEDGMENT}

The authors would like to thank Dr. Bruno Taccardi for his invaluable help and suggestions, and Jayne Davis, Bruce Steadman, and Phil Ershler for expert technical assistance in the experimental studies. This research would not have been possible without the generous support of the Whitaker Foundation, National Institutes of Health BISTI Grant P20 HL68566-01, the Nora Eccles Treadwell Foundation, and the Richard A. and Nora Eccles Harrison Fund for Cardiovascular Research.

\section{REFERENCES}

[1] B. Hopenfeld, J. Stinstra, and R. Macleod, "A mechanism for st depression associated with contiguous subendocardial ischemia," Submitted to J Cardiovasc Electrophysiol, 2004.

[2] J. Stinstra, B. Hopenfeld, and R. Macleod, "Using models of the passive cardiac conductivity and full heart anisotropic bidomain to study the epicardial potentials in ischemia," Proceedings of the IEEE Engineering in Medicine and Biology Society 26th Annual International Conference, 2004.

[3] J. Stinstra, B. Hopenfeld, and R. MacLeod, "On the passive cardiac conductivity," submitted to IEEE Trans. on Biomed. Eng., 2004.

[4] P. Nielsen, I. LeGrice, B. Smaill, and P. Hunter, "Mathematical model of geometry and fibrous structure of the heart," Am J Physiol, vol. 29, p. H1365:H1378, 1991.

[5] B. Taccardi, E. Macchi, R. Lux, P. Ershler, S. Spaggiari, S. Baruffi, and Y. Vyhmeister, "Effect of myocardial fiber direction on epicardial potentials." Circ., vol. 90, pp. 3076-3090, 1994.

[6] W. Smith, W. Fleet, T. Johnson, C. Engle, and W. Cascio, "The ib phase of ventricular arrhythmias in ischemic in situ porcine heart is related to changes in cell-to-cell electrical coupling," Circulation, vol. 92, pp. 3051-3060, 1995.

[7] R. MacLeod, B. Taccardi, and R. Lux, "Electrocardiographic mapping in a realistic torso tank preparation," in Proceedings of the IEEE Engineering in Medicine and Biology Society 17th Annual International Conference. IEEE Press, 1995, pp. 245-246.

[8] K. Reimer and R. Jennings, "Myocardial ischemia, hypoxia and infarction," in The Heart and Cardiovascular System, H. Fozzard et al., Eds. New York: Raven Press, 1986, pp. 1133-2101.

[9] R. Guyton, J. McClenathan, G. Newman, and L. Michaelis, "Significance of subendocardial S-T segment elevation caused by coronary stenosis in the dog," Am. J. Cardiol., vol. 40, pp. 373-380, 1977. 